\title{
Actualidad de la pedagogía ignaciana en la universidad
}

\author{
Araceli Martínez Arroyo \\ Universidad Iberoamericana Ciudad de México, México \\ araceli.martinez@ibero.mx \\ ORCID: https://orcid.org/0000-0002-3760-7792 \\ https://doi.org/10.48102/didac.2022..79_ENE-JUN.95 \\ 8
}

La publicación de este número 79 de la revista DIDAC destinado a la reflexión sobre la actualidad de la pedagogía ignaciana en la universidad, ocurre en el marco de la celebración del Año Ignaciano que conmemora los 500 años de la conversión de san Ignacio de Loyola. Ignatius 500 comenzó a celebrarse en todo el mundo el 20 de mayo de 2021 y concluye el 31 de julio de 2022. La primera fecha corresponde con el aniversario 500 de su herida en Pamplona y, la segunda, con el aniversario 400 de la fecha establecida para su festividad tras su canonización.

Con una génesis jubilar propia de la Compañía de Jesús, esta celebración alude a cuatro hechos centrales o hitos en la vida de Ignacio (s Digital, 2021):

a) Una herida: en 1521 Navarra es invadida por el ejército francés y en la defensa de Pamplona el, entonces caballero, Ínigo de Loyola recibe una bala de cañón que le destroza la pierna derecha y le deja malherida la izquierda. Este hecho no solamente le obliga a trasladarse a la casa-torre familiar en Loyola y a confinarse durante varios meses para su recuperación, sino que también le plantea una ruptura con sus aspiraciones de ascenso en la corte por sus méritos militares. Si bien Íńigo logró caminar tras varias intervenciones, mantuvo una leve afección en la pierna derecha. b) Un encuentro: en el tiempo de su convalecencia, Ínigo agota la lectura de obras de caballería existentes en la biblioteca familiar. Motivado más por el aburrimiento que por la curiosidad se acerca a una historia de Cristo y a un volumen de vida de santos. Descubre en estas biografías, hechos admirables que desea emular. Toma consciencia que tras el estudio de estos personajes su pensamiento comienza a tomar dos líneas: "las cosas que podría hacer por Dios" y las hazañas que "podría hacer para ganar el favor de su rey o de su dama" (López de Lara, 2018, p. 38). Tras una aparición, visión o imaginación vívida de la Madre de Dios llevando en brazos a Su hijo, Ínigo resuelve su deseo de transformar su vida y orientarlo al servicio de Dios.

c) Un maestro: en el primer paso de su transformación, Íñigo se despide de su familia en Loyola en febrero de 1522 decidido a emprender una vida de peregrinación y penitencia. Se traslada al monasterio de Montserrat - uno de los principales focos de renovación religiosadonde recibe hospedaje por unos días. Su peregrinación tenía un destino claro: Jerusalén; para llegar ahí, Ínigo debía embarcar en Barcelona. Sin embargo, este no contaba con que la ciudad tenía prohibida la entrada de visitantes para evitar el contagio de una enfermedad que por entonces azotaba a la población; decide 
trasladarse al pueblo de Manresa en tanto podía acceder al puerto. Su estancia en Manresa se prolongó por once meses en tanto una serie de complicaciones postergaron su viaje. Durante este periodo, Iñigo transformó su apariencia física y maduró en el descubrimiento y aprendizaje de Dios. Alternó el "entusiasmo, la angustia, la penitencia, la desesperación, las visiones espirituales y el misticismo" (sJ Digital, 2021); pidió limosna cada día, azotó su cuerpo, se alojó en un convento o en un hospicio y se retiró a una cueva a orar. "En ese tiempo le trataba Dios de la misma manera que trata un maestro a un nińo, enseñándole" (Rambla, 2015, p. 71). Fue en este sitio que Ínigo esbozó las notas principales del futuro libro de los Ejercicios espirituales. "Íńigo es ya un maestro de espíritu" (sJ Digital, 2021).

d) Un camino: Antes de llegar al Monasterio de Montserrat y, posteriormente a la ciudad de Manresa (ambas en Barcelona), Ínigo cruzó por distintas localidades de las actuales comunidades del País Vasco, La Rioja, Navarra y Aragón en un itinerario de aproximadamente 650 kilómetros, trayecto que hoy en día es conocido como el "Camino ignaciano". Este hecho se convirtió en un hito de la espiritualidad ignaciana en tanto constituye un modo de situarse en la realidad concreta y en la relación con lo trascendente. Plantea la idea de ponerse y mantenerse en el camino, de andar voluntariamente hacia el encuentro personal con lo trascendente, despojándose y renovándose constantemente. Camino como proceso, como disposición a la transformación.

Como es posible observar, el contexto de esta celebración plantea a quienes comparten la fe cristiana, una invitación a "hacer el camino espiritual de todo converso que va a la búsqueda de Dios" y que permite "al Señor obrar en nuestra transformación inspirados en la experiencia personal de Ignacio" (Sosa, 2019a).

Por su parte, para quienes, desde otras perspectivas, cosmovisiones o credos no comparten esta fe cristiana, esta celebración resulta una invitación a pensarse en un momento como el que vivimos desde estas metáforas clave: herida, confinamiento, transformación, renovación del sentido, del camino y del trayecto personal. Es innegable que en un contexto global como el que aún nos cimbra ante la continuación de la pandemia y el descubrimiento de nuevas variantes detectadas de Covid-19, las respuestas de sentido que podamos recibir son indispensables. "No se trata de voltear hacia atrás, ni de exaltar la figura de Ignacio de Loyola” (Sosa, 2021b), se trata de que, motivados por una experiencia personal, "miremos al pasado para inspirar nuestro presente y renovar nuestra esperanza en el futuro" (Sosa, 2021a).

En este tenor, la celebración Ignatius 500 a la que DIDAC se suma adquiere relevancia no únicamente entre los colegios jesuitas, sino entre todas aquellas instituciones educativas que, interpeladas por los tiempos que corren y los impactos que deja la pandemia, hacen una pausa para discernir y renovar el compromiso con sus ideales. Este número sobre la actualidad de la pedagogía ignaciana en la universidad es un motivo ideal para dicho ejercicio a la luz de este referente en particular.

\section{Pedagogía ignaciana en educación superior}

La labor educativa de la Compañía de Jesús es considerada una tradición viva en permanente proceso de renovación para responder a los nuevos contextos. De acuerdo con el P. General Arturo Sosa (2019a), esta idea de permanente renovación (asociada al hito del camino, previamente expuesto) plantea dos enormes desafíos a los actuales educadores: por un lado, recibir esta tradición educativa (condensada, principalmente, en sus documentos y en otras fuentes); y, por otro lado, asegurar que ésta siga siendo una tradición viva, de ser posible, una pedagogía pertinente y relevante:

[Esta pedagogía] será pertinente en cuanto asuma las realidades culturales que el mundo está desarrollando y los retos que ellas encierran. 
[Será relevante para el presente y el futuro] tanto cuanto esta pedagogía ayude a las nuevas generaciones a construir un mundo más humano, más justo y respetuoso del entorno. (Sosa, 2019a, pp. 9-10).

En relación con las fuentes constituyentes de esta tradición educativa, Martins Lopes (2018) precisa que

consideramos como fuentes de la pedagogía de la Compañía de Jesús la persona de san Ignacio de Loyola, los Ejercicios Espirituales, el modus parisiensis, la Parte IV de las Constituciones de la Compañía de Jesús y las ediciones de la Ratio Studiorum. Hoy en día estas fuentes continúan expresándose en varios documentos, entre los que destacamos: las Características de la educación de la Compañia de Jesús y la Pedagogía ignaciana: Un planteamiento práctico" (p. 36).

A estos dos últimos textos enunciados por Martins, Sosa (2020) añade Colegios Jesuitas: Una tradición viva en el siglo 21. Un ejercicio de discernimiento, y apunta que estos tres documentos se complemen$\tan y$ "deben tomarse juntos como el marco contemporáneo de la educación jesuita" (p. 6).

Destaca el hecho de que estas ocho fuentes son de naturaleza distinta y que el conocimiento de cada una entrańa una experiencia particular.

Con respecto de las tres primeras fuentes señaladas por Martins Lopes (2018) vale la pena formular algunos apuntes y reflexiones:

- la persona de san Ignacio de Loyola: de manera análoga a la celebración Ignatius 500 y los hitos de la vida de san Ignacio que la motivan, la persona y la vida del santo se convierte en fuente de inspiración que permea todas las actividades de la Compañía no tanto por los resultados que Ignacio alcanzó con sus obras sino por el modo de proceder que lo caracterizó, el cual estuvo cimentado en el discernimiento que conduce al bien mayor o para la mayor gloria de Dios. Ad maiorem Dei gloriam, es una divisa sustantiva de la Compañía de Jesús.

- los Ejercicios Espirituales: constituye la obra central de Ignacio de Loyola en tanto, como su nombre lo indica, postulan una ejercitación de la espiritualidad. En palabras de su autor, son "un modo de examinar la conciencia, de meditar, de contemplar, de orar vocal y mentalmente [así como] de otras actividades espirituales...” (Loyola, 2017, p. 11). Si bien los Ejercicios espirituales no abordan al hecho educativo en sí mismo, sí constituyen una clara muestra de un manual práctico y didáctico para la formación espiritual. Su estructura pautada en cuatro semanas, la precisión de las anotaciones al ejercitante y al que da los ejercicios así como la descripción detallada de los momentos y periodos de dichos ejercicios, permiten que su metodología de la experiencia activa trascienda el ámbito de la formación espiritual e inspire otros procesos formativos. Los primeros jesuitas "se dieron cuenta de la eficacia de este método pedagógico que vivenciaban, pues las etapas propuestas para el itinerario se adaptaban a la realidad existencial de cada uno de ellos respetando su ritmo de elaboración y apropiación personal" (Klein, 2002, p. 30).

- el modus parisiensis: se refiere al sistema de enseñanza parisino que san Ignacio recibió en su paso por la Ciudad de la Luz y su formación en los colegios de Montaigu, Santa Bárbara y Saint-Jaques entre 1528 y 1535. Dicho sistema "no era entonces particularmente original, ni estaba consignado en un documento especial [sino que] recogía el producto de una larga experiencia educativa, fraguada en los debates entre humanistas y escolásticos desde el siglo xv" (Klein, 2002, p. 27). El modus parisiensis planteaba una "«arquitectura» educativa que estaba constituida de cuatro elementos: organización, metodología, atención personalizada y formación religiosa” (Klein, 2018, p. 155). La organización consistía en una regulación 
organizacional y curricular graduada y controlada difundida entre todos los miembros de la institución; la metodología hace referencia, entre otras estrategias, a la praelectio o presentación expositiva del docente sobre los textos, las quaestiones de los profesores o alumnos en tornos a los textos y, finalmente, la disputatio, diálogo o debate que redundaba en la apropiación, creatividad y aplicación práctica del conocimiento. La atención personalizada priorizaba el cuidado de la persona. Al estudiante se le entrevistaba ampliamente para situarle en la clase adecuada y, aunque el régimen disciplinar era severo, se recomendaba a los educadores anteponer el diálogo y la exhortación antes que los castigos (Klein, 2018, pp. 159-165). La formación religiosa, por su parte, compren- día numerosas prácticas religiosas habituales (asistir a misa diaria, el sermón dominical, entre otras), pero, por encima de ello lo auténticamente característico de este sistema era el "método que allí estaba en vigor [el cual se caracterizaba] por una actividad infatigable, un ejercicio y una práctica constantes, una especie de gimnasia mental incesante que moviliza el proceso educativo de todos los recursos y todas las facultades de la persona" (Códina, 1968, p. 109 en Klein, 2018, p. 166).

En lo relativo a los documentos que propiamente abordan el tema educativo y que constituyen el fundamento e inspiración de esta pedagogía ignaciana, se enuncian y se describe brevemente su aportación central:

Tabla 1.

Documentos fundantes de la pedagogía ignaciana

Constituciones de la Compañia de Jesús, parte IV (1558)
Los Ejercicios espirituales y las Constituciones son los únicos textos escritos en vida de san Ignacio. Las Constituciones describe las primeras decisiones adoptadas por él sobre la educación de la Compańía, su finalidad, el gobierno de los colegios y universidades, el currículo que se debe adoptar y algunos aspectos metodológicos. En este textos se seńala que, al igual que los colegios, las universidades se aceptan en la Compañía "para la edificación en doctrina y vida no solamente de los nuestros, pero aún más de los de fuera de la Compañía”. (Const. 26/440)
Ratio studiourum

$(1569,1586,1591$ y 1599$)$
A la muerte de san Ignacio en 1556, Diego Laínez le sucedió como general de la Compañía. Fue él quien encargó la preparación de un tratado sobre las normas que habrían de regular a los colegios; la elaboración de este documento fue una promesa que Laínez realizó a san Ignacio. Habrían de redactarse varias versiones de éste antes de llegar a su fórmula definitiva:

- 1569: conocida como Ratio studiourum borgiana -por ser presentada en el generalato de san Francisco de Borja- señalaba lo que debía hacerse en los colegios, pero no decía cómo. Trataba los estudios de Humanidades aunque omitía los referentes a Filosofía y Teología (Mesa, 2019, pp. 131-132).

- 1586: con un detalle de 130 cuestiones, es considerada la primera Ratio. Consta de dos partes: una, sobre la "Selección de opiniones" que habría de fundamentar la enseñanza en la Compañía; otra, sobre el orden que debía seguirse en los estudios: teología, filosofía y humanidades (Mesa, 2019, pp. 132-133). Fue presentada con Claudio Acquaviva como general de la Compañía.

- 1591: contiene "una serie de reglas precisas para las autoridades, para los distintos oficios encargados de las distintas disciplinas y para los estudiantes [y añade un apéndice de] normas particulares para las distintas provincias, y un ejemplo de desarrollo (praelectio) de una clase de Humanidades" (Mesa, 2019, p. 134). Una queja sobre esta versión fue que "no se tuviera en cuenta la situación particular de cada país" (Mesa, 2019, p. 135).

- 1599: es el plan de estudios de los colegios jesuitas. No es un tratado teórico ni una filosofía de la educación. Concebida como una prolongación de la parte IV de las Constituciones, la Ratio atque Institutio Studiorum Societatis Jesu (Método y programa de los estudios de la Compañía de Jesús) descríbe el currículo de la enseńanza de teología, filosofía y humanidades. Klein señala (2002) que este plan equivaldría a la actual enseñanza media (p. 40). La ratio concentra la normativa curricular, didáctica y de gestión escolar. Describe las funciones y tareas del provincial, del rector, del prefecto, de los profesores, de los escolares y de las distintas academias. 
Características de la educación de la Compañía de Jesús (1986)

En celebración del cuarto centenario de la Ratio de 1586, se presenta este trabajo realizado por la Comisión Internacional para el Apostolado de la Educación de la Compañía de Jesús. Características no es considerada una Ratio; es un "documento inspirador [que clarifica las] señas de identidad educativa" (Gil Coria, 2002, p. 248), una declaración de los objetivos en educación que persigue la Compañía con el fin de utilizarla "como un instrumento para progresar en la renovación" (Kolvenbach, P.H., 1986, p. 8). Está dirigida a la educación primaria y secundaria pero también se recomienda a universidades. Realiza un "conexión entre espiritualidad ignaciana y el modo de proceder en educación. La idea de Dios, y de Cristo como modelo, se relacionan con el crecimiento global de la persona. Los conceptos de «magis», discermiento, excelencia, comunidad educativa, dimensión social (educar desde la perspectiva de los pobres), son líneas clave de este documento" (Gil Coria, 2002, p. 248).

Características está dividida en nueve secciones que contienen las 28 características de la educación de la Compañía. Las nueve secciones, a saber, son: Dios, libertad humana, búsqueda de la libertad, Cristo como modelo de persona, la acción, en la iglesia, el "Más" (Magis), la comunidad y el discernimiento. Algunas de las caracterísiticas señaladas son: es un instrumento apostólico; promueve el diálogo entre la fe y la cultura; celebra la fe en la oración personal y comunitaria, en otras formas de culto y en el servicio; sirve a la fe que realiza la justicia; persigue la excelencia en su acción formativa; se realiza en una estructura que promueve la comunidad, entre otras (ICAJE, 1986, pp. 104-110).

Este texto ofrece una pedagogía práctica, una estrategia operativa que corresponde al sentido de identidad y de dirección que planteó Características. Ante la dificultad de formular una nueva Ratio de aplicación universidad que concretara las intenciones de Características, el Consejo Internacional para el Apostolado de la Educación de la Compañía de Jesús resolvió proponer un Paradigma Pedagógico Ignaciano (PPI), que ofreciera "un estilo y proceso didácticos particulares". Este paradigma si bien está pensando en la educación formal, puede tener aplicabilidad en contextos y temporalidades distintas. El pPI se caracteriza por consideras cinco elementos claves del proceso de aprendizaje y del crecimiento personal:

a) Contexto: refiere a los diversos entornos em que ocurre la enseñanza y el aprendizaje (el contexto socioeconómico y cultural), el centro de enseñanza, la situación personal de los miembros de la comunidad educativa, los conocimientos previos del estudiante, la planificación de las actividades de aprendizaje, entre otras.

Pedagogía ignaciana. Un planteamiento práctico (1993) b) Experiencia: es el acercamiento cognoscitivo y afectivo a la realidad. La experiencia provoca emociones en el estudiante.

c) Reflexión: el estudiante integra de manera significativa lo aprendido y profundiza en su reflexión cuestionando la relación con uno mismo, con los demás, y con el entorno del que se forma parte.

d) Acción: la reflexión conduce a acciones interiores (opciones personales internas hacia valores y actitudes) y a acciones exteriores, coherentes con dichas convicciones. La acción implica respetar la libertad de cada uno y animar la decisión de compromiso con el magis.

d) Evaluación: no se refiere propiamente al ejercicio de evaluación académica, sino a "la evaluación periódica del progreso de los estudiantes en sus actitudes, prioridades y acciones acordes con el objetivo de ser una "persona para los demás»" (ICAJE, 1993, p. 20)

Este PPI destaca por la posibilidad de personalizar la enseñanza al tiempo que acentúa la dimensión social de la enseñanza y el aprendizaje.

Este documento, al igual que sus homólogos contemporáneos fue redactado por la Comisión Internacional para el Apostolado de la Educación de la Compañía de Jesús [ICAJE, por sus siglas en inglés] en el actual generalato del P. Arturo Sosa. Colegios Jesuitas "sigue esta tradición de ayudar a nuestro apostolado educativo a reflejar y discernir los desafíos y oportunidades particulares de nuestro tiempo, continuando el proceso necesario de renovación, innovación y re-imaginación, que nuestra educación requiere durante este cambio de época que experimentamos hoy" (Sosa, 2020, p. 5). Además de los documentos fundacionales que se han promulgado recientemente (Características y Pedagogía Ignaciana), este texto toma como base las Preferencias apostólicas Universales de la Compañía de Jesús, 2019-2029 y sintetiza en 10 claves, los identificadores que deben estar presentes en todo colegio jesuita. En ese sentido señala que los colegios jesuitas están comprometidos: 1) a ser católicos y ofrecer formación profunda en la fe en diálogo con otras religiones y visiones del mundo; 2) a crear un ambiente seguro y saludable para todos; 3) con la ciudadanía global; 4) con el cuidado de la creación; 5) con la justicia; 6) a ser accesibles para todos; 7) con la interculturalidad; 8) a ser red global al servicio de la misión; 9) con la excelencia humana, y, 10) con el aprendizaje de por vida.

Fuente: Elaboración propia a partir de Gil Coria (2002), Klein (2002), Lange (2005), Mesa (2019), Sosa (2019a) y las fuentes primarias referidas.
Esta revisión de las fuentes que inspiran la pedagogía ignaciana, aunque escueta, permite dimensionar que esta "histórica y clara apuesta por la educación por parte de la Compañía de Jesús ha sido acompa- ñada de un modelo propio de atender a los retos y necesidades de enseñanza” (Guibert, 2020, p. 11). Dicho modelo, no obstante, va más allá de un mero planteamiento metodológico o de una estrategia didáctica, sino que convierte a esta pedagogía ignacia- 
na en un cuerpo de conocimiento con una herencia histórica y una resignificación personal y colectiva permanente del hecho y la tarea educativa y de su relación con un sentido de fe y justicia social que encuentra su inspiración fundamental en la persona y la espiritualidad de san Ignacio de Loyola.

Es verdad que, "en ningún momento, Ignacio relacionó explícitamente su espiritualidad con los principios básicos de la tarea educativa, más bien aplicó tal espiritualidad a los criterios educativos de forma espontánea (Sauvé, 1981, pp. 13-14 en Lange, 2005, p. 99). De ahí que, como sustento de las directrices pedagógicas "subyace el carisma del mismo Ignacio de Loyola como fuente de la "mística de los centros educativos de la Compañía”. De ahí también que sea posible deducir, por tanto, que el desafío de recibir esta tradición educativa exhorta no solamente al conocimiento de estas múltiples fuentes sino que apela a una experiencia personal en su lectura, significación y concreción, y que dicha experiencia coloca a cada una de las personas de esta comunidad educativo en un camino de búsqueda en total libertad. "Buscar: pues, es la consigna ignaciana. Buscar siempre. Estar siempre en camino” y "comprometerse con proyectos, con programas, con acciones" (Narro, 1998, p. 218). Buscar es "ir más allá de lo adquirido, es la clave de un modelo educativo inspirado en la espiritualidad de Ignacio de Loyola. Quizá, como expresa Narro (1998) "el modelo, es, pues, ir más allá de cualquier modelo" (p. 218).

\section{Conversación sobre la actualidad de la pedagogía ignaciana en la universidad en DIDAC 79}

Los diez artículos que conforman este número 79 ofrecen una muestra significativa de la concreción de este paradigma educativo en diversos ámbitos de la vida universitaria. Los tres artículos de la sección "Fábrica de innovaciones", por ejemplo, dan cuenta de experiencias de extensión universitaria y de la formación para el compromiso social a partir, justamente, de la integración de este Paradigma Pedagógico Ignaciano como sustento de la práctica social y de la práctica docente.
Los artículos de la sección "La educación al microscopio" exponen formas diferenciadas de entrar en conversación con esta forma particular de hacer pedagogía, ya sea a partir de la reflexión personal de un docente (Camacho), de la puesta en diálogo del humanismo jesuita con planteamientos contemporáneos de la ciudadanía mundial formulados por la Unesco (Bermeo), o bien, desde la revisión documental de aquellos elementos fundacionales que constituyen el modelo educativo universitario desde la perspectiva ignaciana (Estrella).

La sección "Dossier" ofrece un invaluable planteamiento de Michael J. Garanzini, S.J., (actual Presidente de la Asociación de Colegios y Universidades Jesuitas) y de Michael Baur (coorganizador del Seminario de Pedagogía Jesuita, de la Universidad de Fordham) sobre las características que deben distinguir actualmente a las universidades jesuitas en su inspiración ante este Paradigma Pedagógico Ignaciano y la urgente necesidad de dar respuesta al contexto actual.

Finalmente, en la sección "¿Qué se está haciendo en la Ibero?” se describe la estrategia de formación docente que se está llevando a cabo en la Universidad Iberoamericana Ciudad de México en el marco de la actualización de sus planes de estudios de licenciatura -denominados Manresa- y que tiene como base, el método pedagógico empleado por Ignacio para la formación: ofrecer un modo general que se concreta en ritmos, estilos y necesidades particulares.

Esta sección cierra con un artículo que sintetiza las reflexiones iniciales de un grupo de académicos

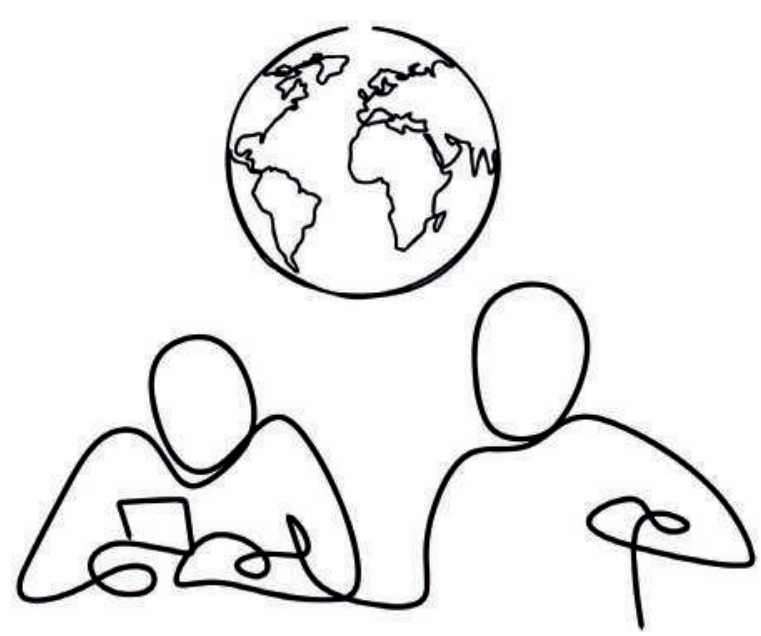

Actualidad de la pedagogía ignaciana en la universidad $\bullet$ Araceli Martínez Arroyo. DIDAC 79 (2021): 2-9 
de esta institución que conversaron en torno a las comprensiones individuales que tienen de la pedagogía ignaciana y de su concreción en las tareas que desarrollan en la institución. El diálogo dejó en evidencia la diversidad de definiciones, énfasis y ejemplos sobre dicha traducción y la perspectiva de un estudio y conversación profunda y prolongada de un tema que todos los participantes consideran vital en su compromiso con el ideario universitario.

\section{Agradecimientos}

En la publicación de este volumen de DIDAC, agradecemos especialmente al Mtro. Juan Carlos Ramírez Robledo y a la Mtra. Georgina Tepale Palma, ambos académicos de tiempo completo de la Universidad Iberoamericana Ciudad de México, por su coordinación de este número y por todas las facilidades otorgadas en cada una de las fases del proceso editorial.

\section{REFERENCIAS}

Amigo, Gustavo, S. J. (tr.) and Álvarez, Daniel, S. J. (rev.) Ratio Studiorum Oficial 1599. Ratio atque Institutio Studiorum Societatis Jesu. https://www.educatemagis.org/es/documents/ ratio-studiorum-oficial-1599/

Comisión Internacional para el Apostolado de la Educación de la Compañía de Jesús [ICAJE]. (1986). Características de la educación de la Compañia de Jesús. Colección Pedagogía Ignaciana, 1. Tlaquepaque: ITESO.

Comisión Internacional para el Apostolado de la Educación de la Jesuita [ICAJE]. (1993). Pedagogía ignaciana. Un planteamiento práctico. Educate magis. https://www.educatemagis. org/es/documents/pedagogia-ignaciana-un-planteamientopractico/

Comisión Internacional para el Apostolado de la Educación Jesuita [ICAJE]. (2020). Colegios Jesuitas: Una tradición viva en el siglo 21. Un ejercicio de discernimiento. Roma: SJ. Educatio. https://www.educatemagis.org/wp-content/uploads/ documents/2019/11/Documento-Una-Tradici\%C3\% B3n-Viva-Lo-Res-200501.pdf

Gil Coria, E. (ed). 2002. La pedagogía de los jesuitas, ayer y hoy. 2 ed. Serie I: Estudios, 75. Madrid: Universidad Pontificia Comillas.

Guibert, J. M. (2020). Para comprender la pedagogía ignaciana. Colección Educación, 18. Bilbao: Mensajero.

Klein, L. F. (2002). Actualidad de la pedagogía jesuita. Trad. Juan Carlos Solís. Colección Pedagogía Ignaciana, 6. Tlaquepaque: ITESO.

Klein, L. F. (2018). El modo pedagógico jesuita: de París, Alcalá y Mesina a nuestros días. Martins Lopes, José Manuel. (2018). La pedagogía de la Compañia de Jesús: Contribuciones para el diálogo. Colección Jesuitas, 9. Ciudad de México: Buena Prensa.

Kolvenbach, P.H. (1986). Carta introductoria del Padre General. En Comisión Internacional para el Apostolado de la Educación de la Compañía de Jesús. Características de la educación de la Compañia de Jesús. Colección Pedagogía Ignaciana, 1. Tlaquepaque: ITESO.

Lange Cruz, W. I. (2005). Carisma ignaciano y mistica de la educación. Serie I: Estudios, 93. Madrid: Universidad Pontificia Comillas.

López de Lara, P. (2018). Iñigo. Una biografía de San Ignacio de Loyola. Ciudad de México: Buena Prensa.

8 - Actualidad de la pedagogía ignaciana en la universidad Araceli Martínez Arroyo. DIDAC 79 (2021): 2-9
Loyola De, San Ignacio. (2017) Ejercicios espirituales. (Texto modernizado por Manuel Iglesias). Colección Jesuitas, 1. Ciudad de México: Buena Prensa.

Martins Lopes, J. M. (2018). La pedagogía de la Compañia de Jesús: Contribuciones para el diálogo. Colección Jesuitas, 9. Ciudad de México: Buena Prensa.

Mesa, J. A. (Ed.) (2019). La pedagogía ignaciana. Textos clásicos y contemporáneos sobre la educación de la Compañia de Jesús desde san Ignacio de Loyola hasta nuestros dias. Colección "Manresa" 70, Bilbao-Madrid: Mensajero-Sal Terrae- Universidad Pontificia Comillas.

Rambla Blanch, J. M. (Ed.) (2015). El peregrino. Autobiografía de san Ignacio de Loyola. 2 ed. Colección Manresa, 2. Bilbao: Mensajero-Sal Terrae-Universidad Pontificia Comillas.

Sauvé, J. (1981). La educación de los Jesuitas y la Espiritualidad Ignaciana. CIS, núm. 38.

SJ Digital. (2021). Ignatius 500. Recuperado de https://ignatius 500.org/es/celebramos

Sosa, A. (2019a). Año Ignaciano 2021-2022. Carta del Padre General. https://www.jesuits.global/sj_files/2019/09/201923_27sept19_ESP.pdf

Sosa, A. (2019b). "Prólogo". Mesa, José Alberto. (Ed.) La pedagogía ignaciana. Textos clásicos y contemporáneos sobre la educación de la Compañia de Jesús desde san Ignacio de Loyola hasta nuestros días. Colección "Manresa" 70, BilbaoMadrid: Mensajero-Sal Terrae-Universidad Pontificia Comillas

Sosa, A. (2020). "Carta 2019/5. Colegios Jesuitas: una tradición viva”, pp. 5-7. Comisión Internacional para el Apostolado de la Educación Jesuita. Colegios Jesuitas: Una tradición viva en el siglo 21. Un ejercicio de discernimiento. Roma: sJ Educatio. https://www.educatemagis.org/wp-content/uplo ads/documents/2019/11/Documento-Una-Tradici\%C3 \%B3n-Viva-Lo-Res-200501.pdf

Sosa, A. (2021a). En camino con Ignacio. Episodio 1. Jesuits Global. https://www.youtube.com/watch?v=tVenA_TZLKs\& feature=youtu.be

Sosa, A. (2021b). Presentación del libro del Padre General "En Camino con Ignacio". https://jesuitas.lat/noticias/14-nivel1/6288-presentacion-del-libro-del-padre-general-en-camino-con-ignacio 


\section{SEMBLANZA}

Doctora en Educación y licenciada en Pedagogía por la Universidad Complutense de Madrid. Maestra y licenciada en Pedagogía por la Universidad Nacional Autónoma de México. Miembro de la Asociación Española de Lectura y Escritura (AELE), cursa la Especialidad en prácticas narrativas en contextos educativos. Su actividad docente ha estado relacionada con asignaturas de filosofía de la educación y diseńo educativo. Se ha desarrollado en actividades de formación docente y diseño curricular en programas de licenciatura y posgrado; en investigación pedagógica sobre temas de utopías educativas y de prácticas sociales de escritura en contextos universitarios, así como en el diseño y edición de contenidos y recursos educativos impresos y digitales. Es académica de tiempo completo adscrita al Programa de Desarrollo e Innovación Docente de la Universidad Iberoamericana Ciudad de México, responsable de la investigación "La dimensión social en el curriculum de la Universidad Iberoamericana" y editora de la revista DIDAC. 